\author{
Aging and Oxidative Stress \\ Guest Editor: Mitsuyoshi Matsuo
}

\title{
Life Span and Oxidative Stress in Nematode
}

\author{
Naoaki Ishii* \\ Department of Molecular Life Science, Tokai University School of Medicine, Isehara, Kanagawa 259-1193, Japan
}

Received 5 September, 2003; Accepted 14 October, 2003

\begin{abstract}
Summary The nematode Caenorhabditis elegans has proven a robust genetic model for studies of aging and the roles of oxidative stress. In this review we focus on the genetics of select long-lived and short-lived mutants of $C$. elegans that have proven useful in revealing the relationships that exist between oxidative stress and life span. The former are known to be controlled by an insulin/insulin-like growth factor (IGF -1)-like pathway, while the latter are affected by mitochondrial functions.
\end{abstract}

Key Words: nematodes, oxidative stress, life span, insulin/IGF-1-like signal pathway, mitochondria

\section{Introduction}

Aging is controlled by a complex interplay of genetic and environmental factors. In common aging, one of the most interesting relationships to emerge from aging research is that the product of the standard metabolic rate and the maximum life span is roughly constant for various animals. Thus, animals with lower standard metabolic rates generally live longer than animals with higher rates [1]. This concept, called the Lifespan Energy Potential (LEP), suggests that metabolic rate is closely related to aging. Metabolic rates can be experimentally manipulated. For example caloric restriction extends life span in rodents, nematodes, yeast and probably primates [2-7]. The mechanism is still unclear, but one hypothesis is that caloric restriction slows energy metabolism. Related to these studies is the positive

\footnotetext{
* To whom correspondence should be addressed.

Tel: +81-463-93-1121 ext. 2650 Fax: +81-463-94-8884

E-mail: nishii@is.icc.u-tokai.ac.jp
}

correlation between antioxidant concentrations in the tissues of mammalian species and their maximum lifespan potential (MLSP) [1]. Reactive oxygen species (ROS) such as the superoxide anion $\left(\mathrm{O}_{2}^{-}\right)$, hydrogen peroxide $\left(\mathrm{H}_{2} \mathrm{O}_{2}\right)$, and the hydroxyl radical $(\cdot \mathrm{OH})$ are produced as byproducts of energy metabolism. Such endogenously generated molecules can readily attack a wide variety of cellular entities, resulting in damage that compromises cell integrity and function $[8,9]$. This can cause or at least contribute to a variety of pathologies, including some in humans [10]. To lessen the consequences of this damage, cells have evolved complex defense mechanisms, including enzymes, e.g., superoxide dismutase (SOD) and catalase as well as various non-enzymatic antioxidants, e.g., vitamins $\mathrm{C}$ and $\mathrm{E}$ and glutathione that act to detoxify the offending molecules $[9,11$, 12]. Tolmasoff et al. found that the ratio of SOD specific activity to specific metabolic rate increased with increasing MLSP for all species examined [13]. These data show an important correlation between life span and energy metabolism as well as between oxidative stress and antioxidant defense. 
To learn about the genes that affect aging, the use of animals with established genetics is essential. This is a limited list (e.g. human, mice, fruit fries and so on). One of these animals, the free-living nematode Caenorbabditis elegans (C. elegans), has been employed widely to explore the genetics of aging. One extremely successful approach has been to isolate mutants that extend life span. Conversely, genetic analyses of mutants that shorten life span have also provided insights into aging as it occurs in wild type. In this review, we discuss insights provided by the genetic analysis of long- and short-lived mutants that are affected by insulin-like signaling and mitochondrial functions in energy metabolism, respectively.

\section{Caenorhabditis elegans as a Model System for Aging Research}

C. elegans can be readily cultured in petri plates on a simple diet of Escherichia coli and reproduces with a rapid life cycle of approximately 3.5 days at $20^{\circ} \mathrm{C}$. Embryonic development is rapid, taking only $13 \mathrm{~h}$ at $20^{\circ} \mathrm{C}[14,15]$. The cell lineage has been traced from single-celled zygote to adult and the entire cell lineage has been determined [14-17]. The first half of embryogenesis is characterized by extensive and rapid cell divisions, with embryos reaching 558 cells after $5 \mathrm{~h}$. During the second half, cell divisions cease and the body elongates. After hatching, larval development proceeds through four molts, which punctuate larval stages L1 through L4. About $10 \%$ of the cells in the first larval stage undergo further cell divisions contributing to the hypodermis, neurons, musculature, and somatic gonadal structures. This culminates in adults that contain fewer than 1,000 somatic cells and have been generated from a largely invariant set of cell divisions. Under adverse environmental conditions such as a high population density or starvation, larvae develop into an arrested and quiescent stage called dauer larvae [18]. Dauer larvae consume energy but do not feed, and their metabolism differs markedly from all other stages [19].

C. elegans offers several distinct advantages for aging research, one of which is a short maximum life span of approximately 30 days. In addition, dauer larvae can easily survive for over three months with no effect on post-dauer life span, and, as such, have been described as non-aging [20]. As described below, the genetics of dauer formation overlaps with that of adult life span, mitochondrial functions, and oxidative stress responses. Genetic approaches have proven useful for identifying the genes and pathways that regulate aging [21]. In part this derives from its self-fertilizing, hermaphroditic mode of reproduction, which facilitates mutant isolation (http: //www. wormbase.org/). Literally thousands of mutants have been isolated, each affecting virtually any all biological processes including aging [22]. This classical approach is complemented by some powerful molecular genetics. For example, C. elegans holds the distinction of being the first metazoan to have its genome completely sequenced. In addition, germline transformation is readily accomplished through microinjection, enabling the creation of transgenics, including those with reporter genes. C. elegans has also proven susceptible to the phenomena of RNAi. By exposing animals to double-stranded RNA, an organismal response is triggered that mimics the null phenotype of the gene corresponding to that particular RNA [23]. Because all putative genes have been identified in silico, RNAi provides the opportunity to at least crudely determine the phenotypes resulting from inactivation of literally every C. elegans gene. In addition to these advantages, a soma consisting of fewer than 1,000 cells, all of which are postmitotic in adults, offers the opportunity to detect cumulative age-related cellular alterations $[24,25]$. In addition to these biological advantages, there exists a general spirit of cooperativity and free exchange of information within the "worm community." For example the Caenorbabditis Genetics Center at the University of Minnesota distributes a large number of strains free of charge. Finally, there are a number of Internet sites (most notably http://elegans.swmed.edu) that serve as excellent resources.

\section{Regulation of Aging and Oxidative Stress by the Insulin/IGF-1-like Signaling Pathway}

Studies in C. elegans have led to the identification of genes that have major effects on life span. These genes associate with the insulin/IGF-1-like signaling pathway. For example, age-1 and daf-2 extend life span by up to $100 \%[26,27]$. This increased life span is known as the Age phenotype. The daf-2 gene encodes a protein of the mammalian insulin/IGF-1 like receptor and growth factor receptor [28]. DAF2 activates a conserved phosphatidylinositol-3-OH kinase (PI3K)/3-phosphoinsitide-dependent kinase1 (PDK1)/Akt signaling pathway [29-36]. The age1 encodes a catalytic subunit of PI3K [29] and the 
$p d k-1$ gene encodes a PDK1 homologue [30]. Mutations of $d a f-16$ and $d a f-18$ suppress the Age phenotype of age-1 and daf-2 mutations [37-41]. The daf16 gene encodes a forkhead transcription factor [31, 36]. The daf-18 gene encodes a protein similar to the human PTEN protein that has phosphatidylinositol 3,4,5-triphosphate $\left(\mathrm{PIP}_{3}\right)$ 3-phosphatase activity $[33,34]$, thereby possessing antagonist actions to that to the age-1 gene. daf- 2 acts cell non-autonomously and cell lineages included neurons are involved in life span regulation [42]. Wolkow et al. showed that the limiting expression of daf-2 and age1 to neurons is sufficient to cause major effects on longevity [43]. DAF-16 might regulate the genes that are involved in the biosynthesis of a secondary signal that emanates from daf-2-expressing cells. On the other hand, the mutation of $d a f-12$ that encodes an orphan nuclear hormone receptor enhances the longevity of some daf-2 alleles [37, 44]. The longevity induced by germ line ablation requires functional daf-16 and daf-12, suggesting that the gonad also might affect aging [45].

Mutations of components in the insulin/IGF-1like signaling pathway also confer resistance to heavy metals [46], heat stress [47], ultraviolet light (UV) [48], and ROS [49-52]. This suggests that the life span extension results from the coordinate over expression of stress-response genes [49]. daf-16 mutation also suppresses the phenotype. Insulin/ IGF-1-like signaling is thought to lead to phosphorylation of DAF-16 by AKT activity. DAF-2 pathway prevents DAF-16 accumulation in nuclei [53]. It is known that the DAF-16 protein is located in cytosol under an atmospheric condition, while it moves to nuclei under hyperoxia [54]. Recently, we also found that, even under an atmospheric condition, the DAF-16 protein remains in nuclei of mev1 mutant as shown below, which has abnormal energy metabolism and then overproduces ROS from mitochondria (unpublished data). These results suggest that energy metabolism is intimately related with oxidative stress and the daf- 16 gene is thought to regulate not only energy metabolism but also resistance to oxidative stress. Lin et al. have shown that both sensory neurons and germline activity regulate DAF-16 accumulation in nuclei, but the nuclear localization patterns are different [53]. This reveals the unexpected complexity of the DAF-16dependent pathway that regulates aging and oxidative stress.

The current state of our understanding is summa-

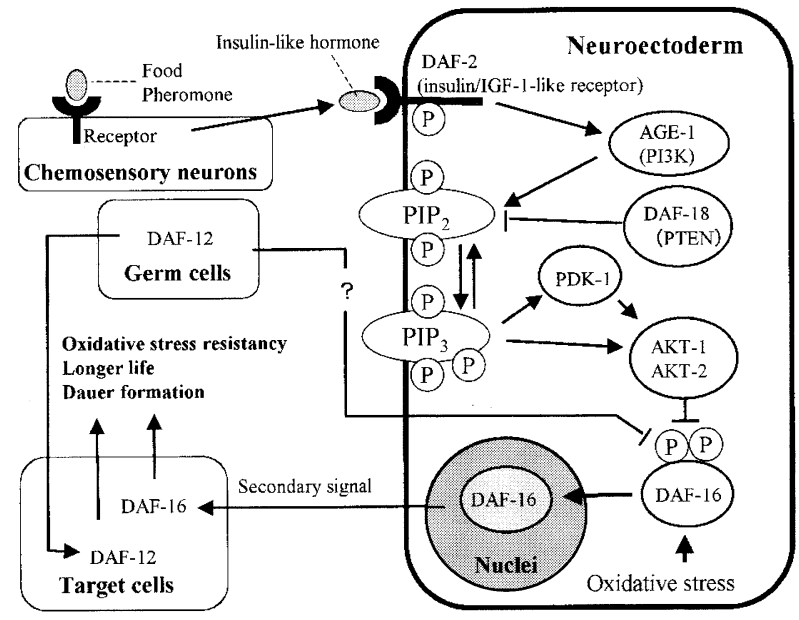

Fig. 1. Insulin/IGF-1-like signaling pathway in nematode. $\mathrm{P}$, phosphate, $\mathrm{PIP}_{2}$, phosphatidylinositol 3,4-diphosphate; $\mathrm{PIP}_{3}$, phosphatidylinositol 3,4,5-triphosphate.

rized by Fig. 1.

\section{Mitochondrial Contribution to Aging and Oxida- tive Stress}

Many short-lived mutants from C. elegans have been isolated. It has been claimed upon occasion that short-lived mutants may not provide significant insights into the normal aging process. This concern is founded on the premise that, at least theoretically, mutations in a large number of genes can negatively impact life span in ways that do not contribute to normal aging. However, molecular characterization of short-lived C. elegans mutants (e.g., mev-1 and gas-1) indicates that this reservation has not been realized. Because these mutants exhibit normal patterns of aging, except at accelerated rates, and because they are affected by a known determinant of aging in wild-type organisms, namely oxidative stress, it seems reasonable to assume that their study will illuminate mechanisms important in normal aging as opposed to aberrant processes irrelevant to wild-type organisms.

The mev-1 and gas-1 genes encode different subunits in electron transport complexes in mitochondria. The energy metabolism in aerobic organisms is almost exclusively the result of glycolysis, the Krebs cycle and electron transport. With respect to electron transport, five membrane-bound complexes within mitochondria form the respiratory chain that sequentially transfers electrons through a series of 
donor/acceptors, with $\mathrm{O}_{2}$ as the final acceptor. Electrons enter the electron transport system through either complex I (NADH-CoQ oxidoreductase) or complex II (succinate-CoQ oxidoreductase), which are capable of using metabolic products of the Krebs cycle to start the flow of electrons in the respiratory chain. The electrons are transferred via two, singleelectron reductions to $\mathrm{CoQ}$ (Coenzyme $\mathrm{Q}$ or ubiquinone), thereby reducing CoQ first to ubisemiquinone and then to ubiquinol (dihydroubiquinone). At complex III $\left(\mathrm{CoQH}_{2}\right.$-cytochrome $c$ oxidoreductase), ubiquinol undergos two sequential and spatially separate one-electron oxidations, a process called the $\mathrm{Q}$ cycle. These reducing equivalents are then transferred to cytochrome $c$ and complex IV (cytochrome $c$ oxidase). In the final step in the process, complex V (ATP synthase) synthesizes ATP.

Mutations in gas-1 were isolated based upon their ability to confer hypersensitivity to volatile anesthetics such as halothane or diethyl ether [55]. We have recently determined that gas-1 mutants are hypersensitive to hyperoxia in a temperature-dependent fashion and have a shorter life span. The gas-1 encodes a subunit $(\mathrm{Ip} 49 \mathrm{kDa})$ of complex I of the electron transport system [56]. The mev-1 mutant was isolated based upon its hypersensitivity to the superoxide-generating chemical methyl viologen [57]. In addition to its methyl viologen hypersensitivity, mev-1 mutants are oxygen-hypersensitive with respect to its effects on both development and aging. The mev- 1 mutation causes a decrease in life span depending on oxygen concentration [58]. In addition, mev-1 mutants accumulate fluorescent materials and protein-carbonyl derivatives at significantly higher rates than do wild-type cohorts [24, 25]. Thus, the aging process in mev-1 animals approximates that of wild type except for its precocious nature. The mer-1 mutation has been identified as residing in the putative gene $c y t-1$, which is homologous to succinate dehydrogenase $(\mathrm{SDH})$ cytochrome $b_{560}$ in complex II [59]. This results in a greater than $80 \%$ reduction in complex II activity in the mitochondrial membrane fraction. Complex II, which catalyzes electron transport from succinate to ubiquinone, is composed of SDH (a flavoprotein: Fp and iron-sulfur protein: Ip) and two other subunits (small and large subunits of cytochrome $b$ as CYT1).

The biochemical pathologies include elevated ROS present in mev-1 mutants. Specifically, $\mathrm{O}_{2}{ }^{-}$ levels in both intact mitochondrial and sub-mito- chondrial particles are approximately two times greater in mev-1 mutants than in the wild-type. The electron transport system is also the major endogenous source of ROS. It has been estimated that generation of the $\mathrm{O}_{2}^{-}$and its dismutated product $\mathrm{H}_{2} \mathrm{O}_{2}$ may constitute as much as $1-2 \%$ of total electron flow, although others have placed this value at $0.1 \%$ [60]. It is known that oxygen is initially converted to the $\mathrm{O}_{2}{ }^{-}$by electrons leaked from complex I and mainly complex III [61-63]. This means that the mev-1 mutation either exacerbates $\mathrm{O}_{2}{ }^{-}$production at this location or, in some indirect way, increases $\mathrm{O}_{2}{ }^{-}$ production at another point in electron transport, perhaps even at complex II.

A second important biochemical pathology is that of reduced glutathione (GSH) concentration in mev1 animals. GSH is an important ROS scavenger, and its reduced levels in mev-1 likely exacerbates the deleterious consequences of increased $\mathrm{O}_{2}{ }^{-}$production described immediately above. Third, a number of biochemical pathologies likely derive from the role played by SDH in the Kreb's cycle. 1) The ratio of lactate to pyruvate is significantly higher in mev-1 mutants, suggesting that a metabolic imbalance known as lactate acidosis occurs in these animals. 2) A number of Kreb's cycle intermediates are present at abnormal concentrations in mev-1 mutants. Finally, a loss of mitochondrial membrane potential $\left(\Delta \psi_{m}\right)$ and alteration of CED-9 and CYT-1 protein levels under hyperoxia were observed [64]. As a result, ultra-structural abnormalities of mitochondria have been noted in mev-1 animals, particularly those reared under hyperoxic conditions. The abnormality causes apoptosis or programmed cell-death, in C. elegans. Hyperoxia induces massive apoptosis in mev-1 animals, particularly in embryos. Even under atmospheric oxygen apoptosis is significantly elevated in a mev-1 genetic background. In all likelihood these cellular pathologies are precipitated under the increased oxidative stress, biochemically manifested as both increased ROS and reduced GSH as well as the various metabolic imbalances. Figure 2 shows the summary of the pleiotropic phenotypes of mev-1.

At the organismal level, mev-1 mutants are hypersensitive to oxidative stress in terms of both development and aging. In addition, the developmental hypersensitivity and life span shortning are likely, at least in part, due to the supernumerary apoptosis. Finally, the observation that $\mathrm{SOD} /$ catalase mimetics can extend the life span of mev-1 to wild-type duration provides further evidence of the important role 


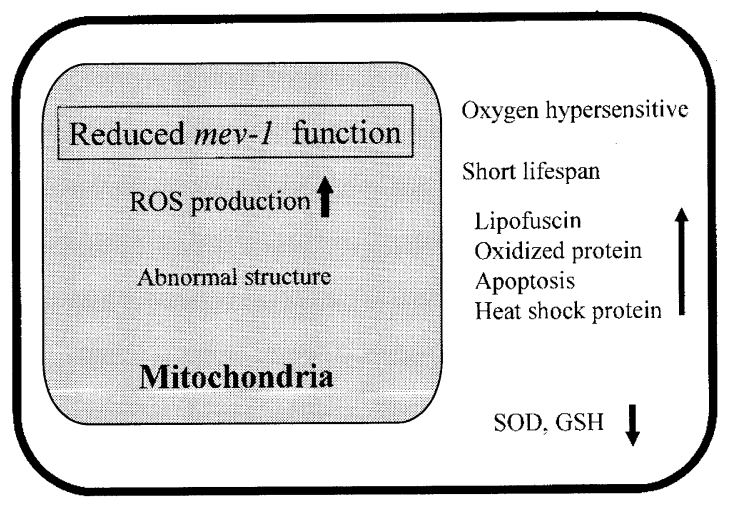

Fig. 2. Summary of the pleiotropic phenotypes caused by the mev-1 mutation.

of ROS and their sequelae in determining organismal life span [65].

On the other hand, in addition of longer life span, mutations in $c l k-1$ exhibit pleiotropic phenotypes that have a temporal component, including in the cell cycle, embryonic development, postembryonic development, behavioral rhythms (e.g., swimming, pharyngeal pumping, and defecation) and fertility [66]. The clk-1 gene has been characterized and found to be conserved among eukaryotes, including humans and rodents, and was identified as a homologue of the gene of Saccharomyces cerevisiae COQ7/ CAT5 located in the inner membrane of yeast mitochondria [67]. The COQ7/CAT5 is necessary for the biosynthesis of CoQ in yeast. As CoQ acts in electron transport, fatty acid oxidation, and uridine synthesis, yeast coq7/cat5 mutants, which lack $\mathrm{CoQ}_{6}$, are unable to grow on nonfermentable carbon sources. In contrast to the situation in yeast, which is defective in respiratory growth, C. elegans clk-1 mutants are able to respire almost normally. In fact, the metabolic capacities and the ATP levels of adult clk-1 mutants are unchanged or even higher than those of the wild type [68], and the mitochondria of clk-1 mutants exhibit succinate-cytochrome $c$ reductase activity that is comparable with that of wild-type mitochondria [69]. These observations suggest that CLK-1 is not exclusively involved in CoQ biosynthesis in C. elegans. When clk-1 mutants were cultured on an E. coli mutant that lacks $\mathrm{CoQ}$, they displayed early developmental arrest from eggs, or sterility emerging form dauer stage. Provision of CoQreplete E. coli rescued these defects [70]. It was found that $c l k-1$ mutants lack the nematode $\mathrm{CoQ}_{9}$ isoform and instead contain a large amount of a metabolite that is slightly more polar than $\mathrm{CoQ}_{9}$ and have increased levels of $\mathrm{CoQ}_{8}$, the $E$. coli isoform and reodoquinone-9. CoQ could be supplied from wild-type $E$. coli as a food. Recently, we found that: i) CoQ biosynthesis is dramatically altered in $c l k-1$ mutants of which the mitochondria do not possess the detectable levels of $\mathrm{Co}_{9}$, and instead contain a $\mathrm{CoQ}$ biosynthesis intermediate, demethoxy coenzyme Q (DMCoQ 9 ); and ii) $\mathrm{DMCoQ}$ can functionally replace $\mathrm{CoQ}$ to maintain active respiration in $c l k-1$ mutant mitochondria, despite the absence of $\mathrm{CoQ}_{9}[71]$.

How do these biochemical data translate to the organismal phenotype of longer life span? This is likely related to the fact that $c l k-1$ mutants also have an increased resistance to stress induced by UV-irradiation, heat, and oxidative stress [48]. This implies that the chemical properties of the $\mathrm{Q}$ semiquinone produced from $\mathrm{DMCoQ}$, reduce $\mathrm{O}_{2}{ }^{-}$concentrations and consequently oxidative stress. This is supported by the recent observation by Stenmark et al. [72] that the $c l k-1$ gene encodes a DMCoQ hydroxylase.

\section{Conclusion}

There are many exogenous and endogenous factors that affect aging. In these, ROS is thought to be an extremely important factor. ROS are continuously produced in living organs as byproducts of normal energy metabolism. It is worth emphasizing that the majority of mutations in C. elegans that affect life span, including those that lengthen it, probably do so by modulating oxidative stress. We have presented evidence that both gas-1 and mev-1 mutants are hypersensitive to oxidative stress, which likely contributes to or is a primary cause of their precocious aging. Both mutants are characterized by $\mathrm{O}_{2}{ }^{-}$overproduction in mitochondria and hypersensitivity to oxidative stress. Conversely, most long-lived mutants appear significantly more resistant to oxidative stress than the wild type. Moreover, a strong inverse correlation has been demonstrated between life span and metabolic rate in long-lived mutants of $C$. elegans [73]. Thus, it appears that the mutations that lower metabolism confer longevity probably due to reducing oxidative stress. Given the central role of energy metabolism including mitochondrial functions in perpetrating oxidative stress, it seems clear that the mutants such as age-1, daf-2, and mer-1 will continue to yield insightful views into the mechanism of aging. 


\section{References}

[1] Cutler, R.G.: Antioxidants and longevity of mammalian species, in Molecular Biology of Aging, ed. by Woodhead, A.D., Blackett, A.D., and Hollaender, A., Plenum Press, New York and London, pp. 15-73, 1985.

[2] Johnson, T.E., Mitchell, D.C., Kline, S., Kemal, R., and Foy, J.: Arresting development arrests aging in the nematode Caenorbabditis elegans. Mech. Ageing Dev., 28, 23-40, 1984.

[3] Yu, B.P.: Modulation of Aging Process by Dietary Restriction, CRC Press, Florida, 1994.

[4] Sohal, R.S. and Weindruch, R.: Oxidative stress, caloric restriction, and aging. Science, 273, 59-63, 1996.

[5] Masoro, E.J.: Caloric restriction. Aging, 10, 173-174, 1998.

[6] Roth, G.S., Ingram, D.K., and Lane, M.A.: Calorie restriction in primates; will it work and how will we know ? J. Am. Geriatr. Soc., 47, 896-903, 1999.

[7] Lin, S.J., Kaeberlein, M., Andalis, A.A., Sturtz, L.A., Defossez, P.A., Culotta, V.C., Fink, G.R., and Guarente, L.: Calorie restriction extends Saccharomyces cerevisae life span by increasing respiration. Nature, 418, 344-348, 2002.

[8] Vuillaume, M.: Reduced oxygen species, mutation, induction and cancer initiation. Mutat. Res., 186, 4372, 1987.

[9] Collins, A.R., Duthie, S.J., Fillion, L., Gedik, C.M., Vaughan, N., and Wood, S.G.: Oxidative DNA damage in human cells: the influence of antioxidants and DNA repair. Biochem. Soc. Trans., 25, 326-331, 1997.

[10] Cross, C.E., Halliwell, B., Borish, E.T., Pryor, W.A., Ames, B.N., Saul, R.L., McCord, J.M., and Harman, D.: Oxygen radicals and diseases. Ann. Int. Med., 107, 526-545, 1987.

[11] Fridovich, I.: The biology of oxygen radicals. Science, 201, 875-880, 1978.

[12] Storz, G., Tartaglia, L.A., and Ames, B.N.: Transcriptional regulator of oxidative stress-inducible genes: direct activation by oxidation. Science, 248, 189-194, 1990.

[13] Tolmasoff, J., Ono, T., and Cutler, R.G.: Superoxide dismutase: Correlation with life-span and specific metabolic rate in primate species. Proc. Natl. Acad. Sci. USA, 77, 2777-2781, 1980.

[14] Sulston, J.E.: Cell lineage, in The Nematode Caenorhabditis elelgans, ed. by Wood, W.B., Cold Spring Harbor Laboratory, New York, pp. 123-155, 1988.

[15] Wood, W.B.: Embryology, in The Nematode Caenorhabditis elegans, ed. by Wood, W.B., Cold Spring Harbor Laboratory, New York, pp. 215-241, 1998.

[16] Sulston, J.E. and Horvitz, H.R.: Post embryonic cell lineages of the nematode Caenorhabditis elegans. Dev. Biol., 56, 110-156, 1977.

[17] Sulston, J.E., Schiernberg, E., White, J.G., and Thomson, J.N.: The embryonic cell lineage of the nematode
Caenorhabditis elegans. Dev. Biol., 100, 164-119, 1983.

[18] Cassada, R.C. and Russell, R.L.: The dauer larva, a post-embryonic developmental variant of the nematode Caenorhabditis elegans. Dev. Biol., 46, 326-342, 1975.

[19] Wadsworth, W.G. and Riddle, D.L.: Developmental regulation of energy metabolism in Caenorhabditis elegans. Dev. Biol., 132, 167-173, 1989.

[20] Klass, M. and Hirsh, D.: Non-ageing developmental variant of Caenorbabditis elegans. Nature, 260, 523-525, 1976.

[21] Guarente, L. and Kenyon, C.: Genetic pathways that regulate ageing in model organisms. Nature, 408, 255262, 2000.

[22] Finkel, T. and Holbrook, N.J.: Oxidants, oxidative stress and the biology of ageing. Nature, 408, 239-247, 2000.

[23] Fraser, A.G., Kamath, R.S., Zipperlen, P., MartinezCampos, M., Sohrmann, M., and Ahringer, J.: Functional genomic analysis of $C$. elegans chromosome I by systematic RNA interference. Nature, 408, 325-330, 2000.

[24] Hosokawa, H., Ishii, N., Ishida, H., Ichimori, K., Nakazawa, H., and Suzuki, K.: Rapid accumulation of fluorescent material with aging in an oxygen-sensitive mutant mev-1 of Caenorbabditis elegans. Mech. Ageing Dev., 74, 161-170, 1994.

[25] Adachi, H., Fujiwara, Y., and Ishii, N.: Effects of oxygen on protein carbonyl and aging in Caenorbabditis elegans mutants with long (age-1) and short (mev-1) life spans. J. Gerontol., 53, B240-244, 1998.

[26] Friedman, D.B. and Johnson, T.E.: Three mutants that extend both mean and maximum life span of the nematode, Caenorhabditis elegans, define the age-1 gene. $J$. Gerontol., 43, B102-109, 1988.

[27] Kenyon, C., Chang, J., Gensch, E., Rudner, A., and Tabtiang, R.: A C. elegans mutant that lives twice as long as wild type. Nature, 366, 461-464, 1993.

[28] Kimura, K.D., Tissenbaum, H.A., Liu, Y., and Ruvkun, G.: daf-2, an insulin receptor-like gene that regulates longevity and diapause in Caenorbabditis elegans. Science, 277, 942-946, 1997.

[29] Morris, J.Z., Tissenbaum, H.A., and Ruvkun, A.: A phosphatidylinositol-3-OH kinase family member regulating longevity and diapause in Caenorhabditis elegans. Nature, 382, 536-539, 1996.

[30] Paradis, S., Ailion, M., Toker, A., Thomas, J.H., and Ruvkun, G.: A PDK1 homolog is necessary and sufficient to transduce AGE-1 PI3 kinase signals that regulate diapause in Caenorhabditis elegans. Genes. Dev., 13, 1438-1452, 1999.

[31] Ogg, S., Paradis, S., Gottlieb, S., Patterson, G.I., Lee, L., Tissenbaum, H.A., and Ruvkun, G.: The fork head transcription factor DAF-16 transduces insulin-like metabolic and longevity signals in C. elegans. Nature, 389, 994-999, 1997.

[32] Paradis, S. and Ruvkun, G.: Caenorhabditis elegans Akt/ $\mathrm{PKB}$ transduces insulin receptor-like signals from 
AGE-1 PI3 kinase to the DAF-16 transcription factor. Genes Dev., 12, 2488-2498, 1998.

[33] Rouault, J.P., Kuwabara, P.E., Sinilnikova, O.M., Duret, L., Thierry-Mieg, D., and Billaud, M.: Regulation of dauer larva development in Caenorbabditis elegans by daf-18, a homologue of the tumour suppressor PTEN. Curr. Biol., 9, 329-332, 1999.

[34] Gil, E.B., Malone Link, E., Liu, L.X., Johnson, C.D., and Lees, J.A.: Regulation of the insulin-like developmental pathway of Caenorhabditis elegans by a homolog. of the PTEN tumor suppressor gene. Proc. Natl. Acad. Sci. U.S.A., 96, 2925-2930, 1999.

[35] Mihaylova, V.T., Borland, C.Z., Manjarrez, L., Stern, M.J., and Sun, H.: The PTEN tumor suppressor homolog in Caenorhabditis elegans regulates longevity and dauer formation in an insulin receptor-like signaling pathway. Proc. Natl. Acad. Sci. USA, 96, 7427-7432, 1999.

[36] Lin, K., Dorman, J.B., Rodan, A., and Kenyon, C.: daf-16: An HNF-3/forkhead family member that can function to double the life-span of Caenorbabditis elegans. Science, 278, 1319-1322, 1997.

[37] Larsen, P.L., Albert, P.S., and Riddle, D.L.: Genes that regulate both development and longevity in Caenorbabditis elegans. Genetics, 139, 1567-1583, 1995.

[38] Malone, E.A., Inoue, T., and Thomas, J.H.: Genetic analysis of the roles of daf-28 and age-1 in regulating Caenorhabditis elegans dauer formation. Genetics, 143, 1193-1205, 1996.

[39] Gottlieb, S. and Ruvkun, G.: daf-2, daf-16 and daf-23: Genetically interacting genes controlling Dauer formation in Caenorbabditis elegans. Genetics, 137, 107-120, 1994.

[40] Dorman, J.B., Albinder, B., Shroyer, T., and Kenyon, C.: The age-1 and daf-2 genes function in a common pathway to control the lifespan of Caenorhabditis elegans. Genetics, 141, 1399-1406, 1995.

[41] Tissenbaum, H.A. and Ruvkun, G.: An insulin-like signaling pathway affects both longevity and reproduction in Caenorhabditis elegans. Genetics, 148, 703-717, 1998.

[42] Apfeld, J. and Kenyon, C.: Cell nonautonomy of C. elegans daf-2 function in the regulation of diapause and life span. Cell, 95, 199-210, 1998.

[43] Wolkow, C.A., Kimura, K.D., Lee, M.S., and Ruvkun, G.: Regulation of C. elegans life-span by insulin like signaling in the nervous system. Science, 290, 147-150, 2000.

[44] Antebi, A., Yeh, W., Tait, D., Hedgecock, E.M., and Riddle, D.: daf-12 encodes a nuclear receptor that regulates the dauer diapause and developmental age in $C$. elegans. Genes Dev., 14, 1512-1527, 2000.

[45] Hsin, H. and Kenyon, C.: Signals from the reproductive system regulate the lifespan of $C$. elegans. Nature, 399, 362-366, 1999.

[46] Barsyte, D., Lovejoy, D.A., and Lithgow, G.J.: Longevity and heavy metal resistance in daf-2 and age-1 long-lived mutants of Caenorbabditis elegans. FASEB J., 15, 627-634, 2001.

[47] Lithgow, G.J., White, T.M., Melov, S., and Johnson, T.E.: Thermotolerance and extended life-span conferred by single-gene mutations and induced by thermal stress. Proc. Natl. Acad. Sci. USA, 92, 7540-7544, 1995.

[48] Murakami, S. and Johnson, T.E.: A genetic pathway conferring life extension and resistance to UV stress in Caenorbabditis elegans. Genetics, 143, 1207-1218, 1996.

[49] Honda, Y. and Honda, S.: The daf-2 gene network for longevity regulates oxidative stress resistance and $\mathrm{Mn}$ superoxide dismutase gene expression in Caenorbabditis elegans. FASEB J., 13, 1385-1393, 1999.

[50] Larsen, P.L.: Aging and resistance to oxidative damage in Caenorhabditis elegans. Proc. Natl. Acad. Sci. USA, 90, 8905-8909, 1993.

[51] Vanfleteren, J.R.: Oxidative stress and ageing in Caenorbabditis elegans. Biochem. J., 292, 605-608, 1993.

[52] Finkel, T. and Holbrook, N.J.: Oxidants, oxidative stress and the biology of ageing. Nature, 408, 239-247, 2000.

[53] Lin, K., Hsin, H., Libina, N., and Kenyon, C.: Regulation of the Caenorbabditis elegans longevity protein DAF-16 by insulin/IGF-1 and germline signaling. Nat. Genet., 28, 139-145, 2001.

[54] Henderson, S.T. and Johnson, T.E.: daf-16 integrates developmental and environmental inputs to mediate aging in the nematode Caenorhabditis elegans. Curr. Biol., 11, 1975-1980, 2001.

[55] Morgan, P.G. and Sedensky, M.M.: Mutations conferring new patterns of sensitivity to volatile anesthetics in Caenorhabditis elegans. Anesthesia, 81, 888-898, 1994.

[56] Kayser, E.B., Morgan, P.G., and Sedensky, M.M.: GAS-1: a mitochondrial protein controls sensitivity to volatile anesthetics in the nematode Caenorbabditis elegans. Anesthesia, 90, 545-554, 1999.

[57] Ishii, N., Takahashi, K., Tomita, S., Keino, T., Honda, S., Yoshino, K., and Suzuki, K.: A methyl viologensensitive mutant of the nematode Caenorbabditis elegans. Mutat. Res., 237, 165-171, 1990.

[58] Honda, S., Ishii, N., Suzuki, K., and Matsuo, M.: Oxygen-dependent perturbation of life span and aging rate in the nematode. J. Gerontol., 48, B57-B61, 1993.

[59] Ishii, N., Fujii, M., Hartman, P.S., Tsuda, M., Yasuda, K., Senoo-Matsuda, N., Yanase, S., Ayusawa, D., and Suzuki, K.: A mutation in succinate dehydrogenase cytochrome $b$ causes oxidative stress and ageing in nematodes. Nature, 394, 694-697, 1998.

[60] Nohl, H. and Hegner, D.: Do mitochondria produce oxygen radicals in vivo? Eur. J. Biochem., 82, 563-567, 1978.

[61] Turrens, J.F.: Superoxide production by the mitochondrial respiratory chain. Biosci. Rep., 17, 3-8, 1997.

[62] Finkel, T. and Holbrook, N.J.: Oxidants, oxidative stress and the biology of ageing. Nature, 408, 239-247, 2000. 
[63] Raha, S. and Robinson, B.H.: Mitochondria, oxygen free radicals, disease and ageing. Trends Biochem. Sci., 25, 502-508, 2000 .

[64] Senoo-Matsuda, N., Hartman, P.S., Akatsuka, A., Yoshimura, S., and Ishii, N.: A complex II defect affects mitochondrial structure, leading to ced-3- and ced-4-dependent apoptosis and aging. J. Biol. Chem., 278, 22031-22036, 2003.

[65] Melov, S., Ravenscroft, J., Malik, S., Gill, M.S., Walker, D.W., Clayton, P.E., Wallace, D.C., Malfroy, B., Doctrow, S.R., and Lithgow, G.J.: Extension of life-span with superoxide dismutase/catalase mimetics. Science, 289, 1567-1569, 2000.

[66] Wong, A., Boutis, P., and Hekimi, S.: Mutations in the clk-1 gene of Caenorhabditis elegans affect developmental and behavioral timing. Genetics, 139, 1247-1259, 1995.

[67] Ewbank, J.J., Barnes, T.M., Lakowski, B., Lussier, M., Bussey, H., and Hekimi, S.: Structural and functional conservation of the Caenorhabditis elegans timing gene clk-1. Science, 275, 980-983, 1997.

[68] Braeckman, B.P., Houthoofd, K., De Vreese, A., and Vanfleteren, J.R.: Apparent uncoupling of energy production and consumption in long-lived Clk mutants of
Caenorhabditis elegans. Curr. Biol., 9, 493-496, 1999.

[69] Felkai, S., Ewbank, J.J., Lemieux, J., Labbe, J.C., Brown, G.G., and Hekimi, S.: CLK-1 controls respiration, behavior and aging in the nematode Caenorbabditis elegans. EMBO J., 18, 1783-1792, 1999.

[70] Jonassen, T., Larsen, P.L., and Clarke, C.F.: A dietary source of coenzyme $\mathrm{Q}$ is essential for growth of longlived Caenorhabditis elegans clk-1 mutants. Proc. Natl. Acad. Sci. USA, 98, 421-426, 2001.

[71] Miyadera, H., Amino, H., Hiraishi, A., Taka, H., Murayama, K., Miyoshi, H., Sakamoto, K., Ishii, N., Hekimi, S., and Kita, K.: Altered quinone biosynthesis in the long-lived clk-1 mutants of Caenorhabditis elegans. J. Biol. Chem., 276, 7713-7716, 2001.

[72] Stenmark, P., Grunler, J., Mattsson, J., Sindelar, P.J., Nordlund, P., and Berthold, D.A.: A new member of the family of di-iron carboxylate proteins. Coq7 (clk-1), a membrane-bound hydroxylase involved in ubiquinone biosynthesis. J. Biol. Chem., 276, 33297-33300, 2001.

[73] Van Voorhies, WA., and Ward, S.: Genetic and environmental conditions that increase longevity in $\mathrm{Cae}$ norhabditis elegans decrease metabolic rate. Proc. Natl. Acad. Sci. USA, 96, 11399-11403, 1999. 